\title{
Parámetros productivos y reproductivos de cuyes (Cavia porcellus) de las líneas Saños y Mantaro
}

\author{
Productive and reproductive parameters of guinea pig (Cavia porcellus) \\ of the Saños and Mantaro lines
}

\author{
Danny Julio Cruz ${ }^{1,4}$, Jorge Passuni Huayta ${ }^{2}$, Flor-Anita Corredor ${ }^{1}$, Mariam Pascual $^{3}$
}

\section{Resumen}

\begin{abstract}
El objetivo del estudio fue determinar los parámetros productivos y reproductivos de las líneas de cuyes Saños y Mantaro, desarrollados en la Estación Experimental Agraria Santa Ana, en la sierra central del Perú. Se analizaron registros de los años 2017 al 2020 de 1773 y 1888 cuyes de la línea Saños y Mantaro, respectivamente. Los parámetros productivos evaluados fueron los pesos al nacimiento (PN), al destete (PD) y a la cuarta (P4), octava (P8) y décimo tercera semana (P13). Los parámetros reproductivos fueron el tamaño de camada (TC) e intervalo entre partos (IP). Los efectos fijos considerados en el modelo fueron la línea genética (L), sexo (S), número de parto (NP), TC y temporada (Tp - trimestres). Se incluyeron covariables como el peso de la madre al parto (PMP); PN, PD, $\mathrm{P} 4$ y $\mathrm{P} 8$ en los parámetros productivos. El efecto animal fue incluido como aleatorio en los modelos de parámetros reproductivos. Se utilizó el procedimiento GLM y GLMM del software R (v. 4.0.2) para determinar los parámetros productivos y reproductivos, respectivamente. Los parámetros productivos de peso vivo para las líneas Saños y Mantaro fueron $153.3 \pm 1.0$ y $150.1 \pm 1.0 \mathrm{~g}$ para $\mathrm{PN}, 289.1 \pm 2.0$ y $291.8 \pm 2.1 \mathrm{~g}$ para $\mathrm{PD}, 407.6 \pm 2.4 \mathrm{y}$ $401.3 \pm 2.9 \mathrm{~g}$ para $\mathrm{P} 4,629.9 \pm 4.4$ y $619.1 \pm 4.5 \mathrm{~g}$ para $\mathrm{P} 8, \mathrm{y} 824.2 \pm 4.7$ y $817.2 \pm 4.9 \mathrm{~g}$ para
\end{abstract}

\footnotetext{
${ }^{1}$ Estación Experimental Agraria Santa Ana, Dirección de Desarrollo Tecnológico Agrario, Instituto Nacional de Innovación Agraria (INIA), Hualahoyo-Saños Grande, El Tambo, Huancayo, Junín, Perú

${ }^{2}$ Estación Experimental Agraria Santa Ana, Instituto Nacional de Innovación Agraria (INIA), Hualahoyo-Saños Grande, El Tambo, Huancayo, Junín, Perú

${ }^{3}$ Programa de Genética y Mejora Animal, Instituto de Investigación y Tecnología Agroalimentarias, Torre Marimón, Caldes de Montbui, Barcelona, España

${ }^{4}$ E-mail: cruzfloredj@gmail.com; https://orcid.org/0000-0003-0849-0051
}

Recibido: 14 de septiembre de 2020

Aceptado para publicación: 20 de marzo de 2021

Publicado: 23 de junio de 2021

CLos autores. Este artículo es publicado por la Rev Inv Vet Perú de la Facultad de Medicina Veterinaria, Universidad Nacional Mayor de San Marcos. Este es un artículo de acceso abierto, distribuido bajo los términos de la licencia Creative Commons Atribución 4.0 Internacional (CC BY 4.0) [https:// creativecommons.org/licenses/by/4.0/deed.es] que permite el uso, distribución y reproducción en cualquier medio, siempre que la obra original sea debidamente citada de su fuente original 
P13, respectivamente Los parámetros reproductivos de las líneas Saños y Mantaro fueron $88.0 \pm 3.9$ y $86.0 \pm 3.8$ días, respectivamente para IP, y $2.60 \pm 0.05$ y $2.64 \pm 0.04$ crías, respectivamente para TC. Se encontraron diferencias significativas $(p<0.05)$, pero sin relevancia biológica para $\mathrm{PN}, \mathrm{P} 4$, y $\mathrm{P} 8$ ajustados por los efectos fijos y respectivas covariables, siendo superior la línea Saños. Hubo diferencias estadísticas $(\mathrm{p}<0.05)$ para el TC, siendo superior en la línea Mantaro, así como diferencias no significativas en el IP ( $p>0.05)$ a favor de la línea Saños en PN, P4, P8 sobre la línea Mantaro, mientras que esta última supera en TC a la línea Saños.

Palabras clave: líneas, cuyes, parámetros productivos, parámetros reproductivos

\section{Abstract}

The aim of this study was to determine the productive and reproductive parameters of the Saños and Mantaro guinea pig lines, developed at the Santa Ana Agrarian Experimental Station, in the central highlands of Peru. Records from the years 2017 to 2020 of 1773 and 1888 guinea pigs of the Saños and Mantaro lines, respectively, were analysed. The productive parameters evaluated were the weights at birth (PN), at weaning (PD) and at the fourth (P4), eighth (P8) and thirteenth week (P13). The reproductive parameters were litter size (TC) and calving interval (IP). The fixed effects considered in the model were genetic line (L), sex (S), calving number (NP), TC and season (Tp - trimesters). Covariates were included such as the mother's weight at delivery (PMP); PN, PD, P4 and P8 in the productive parameters. The animal effect was included as random in the reproductive parameter models. The GLM and GLMM procedures of the R software (v. 4.0.2) were used to determine the productive and reproductive parameters, respectively. The body weight (production parameters) for the Saños and Mantaro lines were $153.3 \pm$ 1.0 and $150.1 \pm 1.0 \mathrm{~g}$ for $\mathrm{PN}, 289.1 \pm 2.0$ and $291.8 \pm 2.1 \mathrm{~g}$ for $\mathrm{PD}, 407.6 \pm 2.4$ and $401.3 \pm 2.9 \mathrm{~g}$ for $\mathrm{P} 4,629.9 \pm 4.4$ and $619.1 \pm 4.5 \mathrm{~g}$ for $\mathrm{P} 8$, and $824.2 \pm 4.7$ and $817.2 \pm 4.9 \mathrm{~g}$ for $\mathrm{P} 13$, respectively The reproductive parameters of the Saños and Mantaro lines were 88.0 \pm 3.9 and $86.0 \pm 3.8$ days calving interval, respectively for IP, and $2.6 \pm 0.05$ and $2.6 \pm 0.04$ offspring, respectively for TC. Significant differences were found $(\mathrm{p}<0.05)$, but not biologically relevant for PN, P4, and P8 adjusted for the fixed effects and respective covariates, the Saños line being superior. There were statistical differences $(p<0.05)$ for the TC, being higher in the Mantaro line; similarly non-significant differences in the PI $(p>0.05)$ in favour of the Saños line in PN, P4, P8 over the Mantaro line, while the latter exceeds the Saños line in TC.

Key words: lines, guinea pigs, productive parameters, reproductive parameters

\section{INTRODUCCIÓN}

La producción de cuyes domésticos Cavia porcellus tiene una gran relevancia en los Andes peruanos por ser considerada como una fuente principal de proteína (Dillard et al., 1972; Quijandria et al., 1983; Pascual et al., 2017), y con una población en aumento (INEI, 2018). Su precocidad, prolificidad, baja exigencia en la alimentación y su aptitud para producir carne hacen que presente un gran potencial productivo (Burgos-Paz et al., 2010).

Los niveles productivos y las características morfológicas y fenotípicas de los animales de esta especie tienden a ser influenciadas por el medio ambiente (Arias et al., 2008; Lobo, 2008; Sánchez-Macías et al., 2018), lo que explica la existencia de ecotipos y líneas con gran potencial producti- 
vo de caracteres de importancia económica. A partir de 1990, la Estación Experimental Agraria Santa Ana (Huancayo, Perú) ha venido desarrollando las líneas de cuyes Saños y Mantaro a partir de ecotipos regionales. Inicialmente se identificó y estabilizó caracteres y rasgos diferentes entre estas dos líneas como el color de pelaje y ojos, presencia de remolino en la cabeza y/o en el lomo y número de dedos en las patas (Reyes, 2015; INIA, Comunicación personal). Desde entonces se pretendió seleccionar a favor de la prolificidad en la línea de cuyes Saños y a favor de la precocidad en la línea de cuyes Mantaro. Ante esto, el objetivo del presente estudio fue determinar las características morfológicas y los parámetros productivos y reproductivos de las líneas Saños y Mantaro.

\section{Materiales y Métodos}

\section{Registros de Producción}

Se recopiló información proveniente de las dos líneas de cuyes desarrolladas en la Estación Experimental Agraria Santa Ana, ubicada en la sierra central del Perú. Se utilizaron registros productivos y reproductivos de 1773 y 1888 cuyes de la línea Saños y Mantaro, respectivamente; con datos desde abril de 2017 hasta marzo de 2020.

El conjunto de datos incluyó registros de peso al nacimiento (PN), peso al destete (PD), peso a la cuarta semana (P4), peso a la octava semana (P8), peso a la décimo tercera semana (P13), sexo (S), tamaño de camada (TC) y temporada (doce trimestres) (Tp). Para las características morfológicas se obtuvieron registros de color de pelaje, remolinos en el pelaje, número de dedos en las extremidades anteriores y posteriores y color de los ojos al nacimiento. Además, se incluyó información del peso de la madre al parto (PMP) y el número de parto (NP) hasta el quinto parto de 343 y 359 cuyes madres de las líneas Saños y Mantaro, respectivamente.

\section{Sistema de Producción}

Ambas líneas fueron criadas bajo el mismo sistema productivo. Los animales son identificados al nacimiento con aretes metálicos y pesados (pesos al nacimiento). A los quince días son destetados, pesados (peso al destete) y colocados en grupos de hasta 15 , según sexo. En la semana 13 (edad de venta), los animales son pesados y seleccionados con base a su peso principalmente, para la reproducción y venta. La reproducción es natural con sistema de empadre continuo; el núcleo reproductor es de 5-7 hembras por macho. Las hembras son puestas a reproducción al alcanzar un peso de $850 \mathrm{~g}$; mientras que los machos a los $1100 \mathrm{~g}$. El tiempo que permanecen los animales en reproducción es de 2 años, o hasta 3 años en casos excepcionales.

La alimentación es a base de rye grass italiano (Lolium multiflorum), alfalfa (Medicago sativa) y trebol rojo (Trifolium pratense); con adiciones ocasionales de maíz amiláceo (Zea maíz L), residuos de cosecha de cebada (Hordeum vulgare) y chala seca de maíz como suplemento. Todo el proceso productivo se lleva a cabo en pozas de madera con dimensiones de $2 \times 1.3 \mathrm{~m}$

\section{Análisis Estadísticos}

Los caracteres morfológicos de color de pelaje, remolinos en el pelaje, número de dedos y color de los ojos al nacimiento fueron descritos mediante frecuencias relativas dentro de cada línea de cuyes. Los parámetros productivos fueron analizados con modelos lineales generalizados (GLM, por sus siglas en inglés) mediante la siguiente ecuación: $Y_{i j k l m n}=\mu+L_{i}+S_{j}+N P_{k}+T C_{l}+T p_{m}+b$ $* P_{i j k l m}^{i j k l m n}+\varepsilon_{i j k l m n}$, donde $Y_{i j k l m n}$ corresponde a los parámetros productivos de $\mathrm{PN}, \mathrm{PD}, \mathrm{P} 4$, $\mathrm{P} 8$, y $\mathrm{P} 13 ; \mu$ es la media general; $L_{i}$ es la línea genética (Saños y Mantaro); $S_{j}$ es el efecto de sexo; $N P_{k}$ es el número de parto (1-5); $T C_{l}$ es el tamaño de camada; $T p_{m}$ es el efecto de temporada (doce trimestres) y $\varepsilon_{i j k l m n}$ 
es el residual. Además, se incluyó la covariable $P_{i j k l m n}$ de acuerdo con el parámetro productivo evaluado. Para los parámetros productivos PN, PD, P4, P8 y P13 se utilizó como covariable PMP, PN, PD, P4 y P8, respectivamente.

Los parámetros reproductivos evaluados fueron el intervalo entre partos y el tamaño de camada. El intervalo entre partos fue evaluado con el uso del modelo lineal generalizado mixto (GLMM) $Y_{i j k}=\mu+L_{i}+$ $N P_{j}+H_{k}+P P_{i j k}+\varepsilon_{i j k}$, donde $Y_{i j k}$ es el intervalo entre partos; $L_{i}$ y $N P_{j}$ son los efectos fijos de la línea genética y número de parto; $\mathrm{H}_{\mathrm{k}}$ es el efecto aleatorio correspondiente a cada hembra; $P P_{i j k}$ es el peso de la madre al momento del parto y $\varepsilon_{i j k}$ es el residual.

Para la evaluación del tamaño de camada de las madres se usó el siguiente modelo: $Y_{i j k}=\mu+L_{i}+N P_{j}+M(H)_{k}+b * P P_{i j k}$ $+\varepsilon_{i j k}$, donde $Y_{i j k}$ donde es el tamaño de camada; $L_{i}$ y $N P_{j}$ son la línea genética y número de parto como efectos fijos; $M(H)_{k}$ es el efecto aleatorio que corresponde a las hembras anidadas dentro de cada macho; $P P_{i j k}$ es el peso de la madre al momento del parto, y $\varepsilon_{i j k}$ es el residual.

Los efectos fijos considerados en los modelos mixtos anteriores fueron los que resultaron estadísticamente significativos en modelos simulados previos. Todos estos análisis estadísticos se desarrollaron con el uso de las librerías lme4, nlme y lsmeans del software estadístico R v. 4.0. 2 (R Core Team, 2020).

\section{Resultados}

En el Cuadro 1 se muestra la estructura de colores de pelaje dentro de cada línea y en la Figura 1 se muestran los dos colores más representativos para cada línea de cuyes. Para la característica del color de pelaje, los cuyes de la línea Saños se caracterizaron porque el 80.6\% (1429) presentó un color de pelaje en el cuerpo entero de color bayo y un $18.8 \%$ (333) un color de pelaje bayo en el cuerpo con variaciones de color blanco grande o pequeño en la cabeza. Todos los cuyes (1773) tuvieron ojos negros y sin remolinos en el pelaje. Asimismo, el 99.9\% (1772) tuvo cuatro dedos en las extremidades anteriores $\mathrm{y}$ tres en las posteriores, mientras que un cuy presentó cuatro dedos en todas las extremidades.

En la línea Mantaro, 93.8\% (1771) de los cuyes presentaron color de pelaje colorado en todo el cuerpo con mancha blanca grande y $2.75 \%$ (52) con mancha blanca pequeña en la frente. El 99.9\% (1886) presentó remolinos en el pelaje a la altura de la frente; $99.9 \%$ (1886) registraron ojos negros y $0.1 \%$ (2) ojos rojos. De otra parte, el 99.5\% (1878) tuvo cuatro dedos en las extremidades anteriores y tres en las posteriores, mientras que $0.53 \%$ (10) de los cuyes presentaron cuatro dedos en las extremidades anteriores y entre cuatro a seis dedos en las extremidades posteriores.

Las diferencias estadísticas de las medias mínimo-cuadráticas de $\mathrm{PN}, \mathrm{PD}, \mathrm{P} 4, \mathrm{P} 8$ y P13 para las líneas de cuyes Saños y Mantaro se muestran en la Figura 2. Los gazapos de la línea Saños tuvieron PN de 153.3 $\pm 1.0 \mathrm{~g}$ en tanto que los de la línea Mantaro pesaron $150.1 \pm 1.0 \mathrm{~g}$, habiendo diferencia significativa $(p<0.05)$, pero de poca relevancia biológica. Para PD no hubo diferencia significativa entre los promedios de las líneas Saños $(289.1 \pm 2.0 \mathrm{~g})$ y Mantaro $(291.8 \pm$ 2.1 g. De otra parte, los cuyes de la línea Saños mostraron pesos de P4 y P8 de 407.6 \pm 2.4 y $629.9 \pm 4.4 \mathrm{~g}$, respectivamente, siendo superiores $(\mathrm{p}<0.05)$ a los pesos de $\mathrm{P} 4 \mathrm{y}$ P8 de la línea Mantaro (401.3 \pm 2.9 y $619.1 \pm$ $4.5 \mathrm{~g}$, respectivamente). Finalmente, los promedios de P13 de los cuyes de la línea Saños y Mantaro fueron $824.2 \pm 4.7$ y $817.2 \pm 4.9 \mathrm{~g}$, respectivamente, sin diferencia significativa entre líneas. 
Cuadro 1. Número y porcentaje de colores de pelaje en las líneas de cuyes Saños y Mantaro (Huancayo, Perú)

\begin{tabular}{llcc}
\hline Línea & \multicolumn{1}{c}{ Color } & $\mathrm{n}$ & $\%$ \\
\hline Saños & Bayo & 1429 & 80.60 \\
& Bayo, blanco grande $^{1}$ & 304 & 17.15 \\
& Bayo, blanco pequeño $^{1}$ & 29 & 1.64 \\
& Blanco y bayo $^{2}$ & 7 & 0.39 \\
& Bayo y negro $^{3}$ & 3 & 0.17 \\
& Bayo y plomo $^{3}$ & 1 & 0.06 \\
Mantaro & Colorado, blanco grande $^{1}$ & 1771 & 93.80 \\
& Colorado, blanco pequeño $^{1}$ & 52 & 2.75 \\
& Blanco y colorado $^{2}$ & 37 & 1.96 \\
& Colorado $^{\text {Bayo, blanco pequeño }}{ }^{1}$ & 25 & 1.32 \\
& Bayo & 3 & 0.16 \\
\hline
\end{tabular}

${ }^{1}$ Presencia del segundo color en la cabeza

${ }^{2}$ Ambos colores en similar proporción en todo el cuerpo

${ }^{3}$ Primer color predominante y segundo color en cualquier parte del cuerpo

El parámetro productivo $\mathrm{PN}$ fue influenciado positivamente por el PMP $(\mathrm{p}<0.05)$; de igual manera, las demás covariables como el PN, PD, P4 y P8 resultaron estadísticamente significativas $(\mathrm{p}<0.05)$. En los cuadros 2 y 3 se muestran los pesos promedios en cada etapa evaluada para las líneas Saños y Mantaro según TC, NP y S. Una tendencia notoria es que los parámetros productivos tienden a bajar en función al incremento del TC $(\mathrm{p}<0.05)$. Además, el PN de los gazapos se incrementa desde el primer hasta el tercer parto $(\mathrm{p}<0.05)$ para luego disminuir progresivamente. Por último, el peso vivo de los machos fue mayor al de las hembras $(\mathrm{p}<0.05)$ respectivamente, siendo 153.4 \pm 1.1 y $150.0 \pm 1.1 \mathrm{~g}$ para $\mathrm{PN}, 291.0 \pm 2.2 \mathrm{y}$ $289.8 \pm 2.2$ g para PD, $405.0 \pm 2.6$ y $403.9 \pm$ 2.6 g para $\mathrm{P} 4,634.6 \pm 4.7$ y $614.6 \pm 4.6 \mathrm{~g}$ para $\mathrm{P} 8$, y $831.5 \pm 4.9$ y $810.4 \pm 5.0$ g para P13. Autores como Chauca (1997), BurgosPaz et al. (2010) y Rodríguez et al. (2013, 2015) discuten a detalle cómo influye el NP, TC o S sobre el PN y PD.
Los parámetros reproductivos se presentan en el Cuadro 4. Los resultados muestran una diferencia relevante, pero no significativa entre intervalo entre partos, siendo de 88.0 días en cuyes de la línea Saños y de 86.01 días en cuyes de la línea Mantaro. Madres con mayor peso al parto presentaron menores intervalos entre partos $(\mathrm{p}<0.05)$. El número de parto no influyó sobre el intervalo entre partos; sin embargo, se observa una tendencia a incrementarse con el número de parto en ambas líneas de cuyes.

El tamaño de camada fue mayor en los cuyes de la línea Mantaro ( $p<0.05)$, sin encontrarse diferencias significativas por el número de parto en ambas líneas. Los cuyes de la línea Saños y Mantaro de primer parto tuvieron $2.44 \pm 0.03$ y $2.41 \pm 0.03$ gazapos, respectivamente, valores que se incrementaron hasta el tercer parto donde alcanzaron $2.69 \pm 0.03$ y $2.73 \pm 0.03$ gazapos por parto, respectivamente. En el cuarto parto el TC 


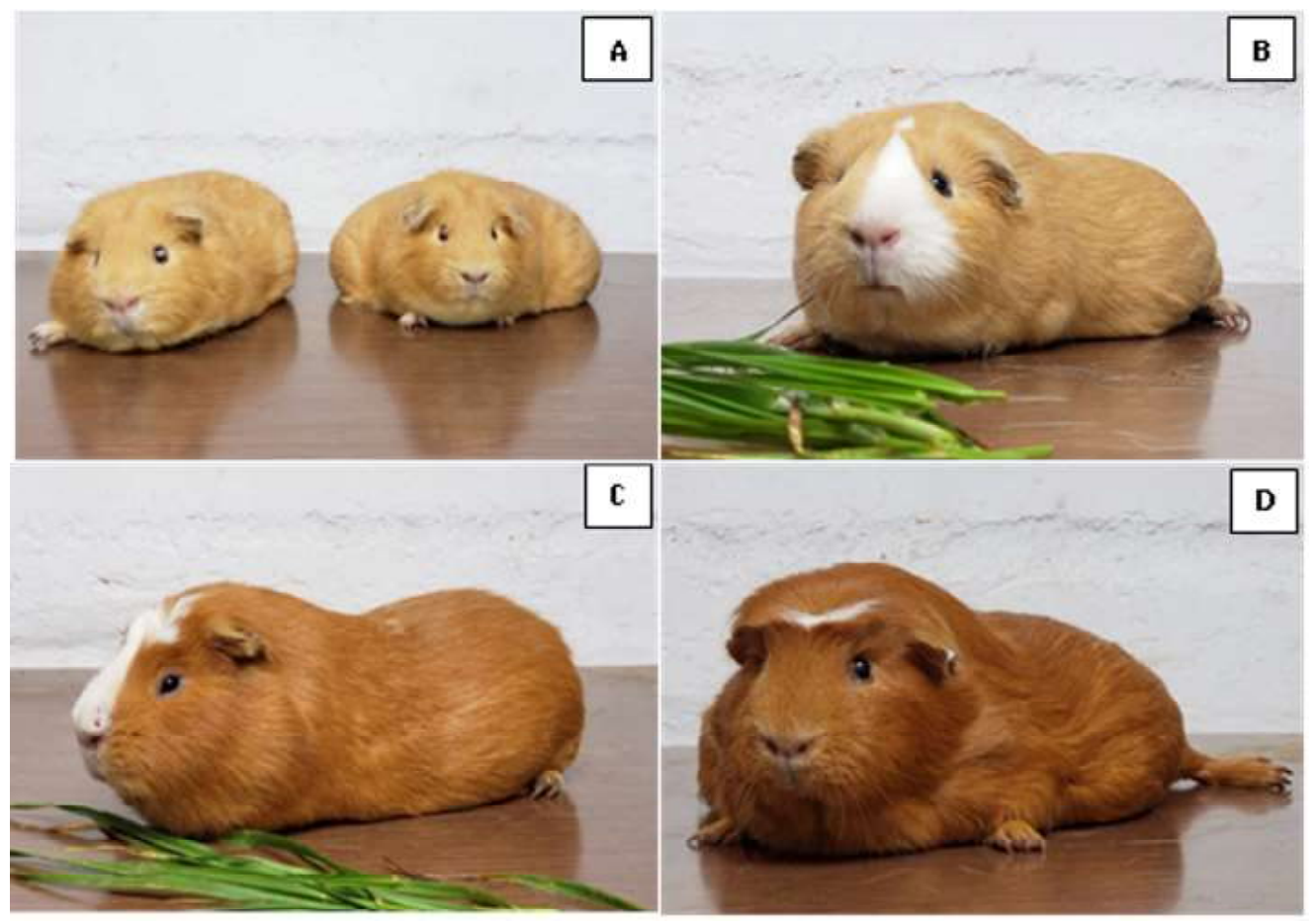

Figura 1. Color de pelaje de cuyes de la línea Saños y Mantaro. A, cuyes Saños de color de pelaje bayo en cuerpo entero. B, cuyes Saños de color de pelaje bayo en el cuerpo y mancha blanca grande en la cabeza. C, cuyes Mantaro de color de pelaje colorado en el cuerpo y mancha blanca grande en la cabeza con remolino a altura de la frente. D, cuyes Mantaro de color de pelaje colorado en el cuerpo y mancha blanca pequeña en la cabeza con remolino a altura de la frente

disminuyó a $2.55 \pm 0.03$ y $2.59 \pm 0.03$, mientras que se incrementó a $2.92 \pm 0.03$ y $2.95 \pm$ 0.03 en el quinto parto en las madres de la línea Saños y Mantaro, respectivamente. Finalmente, se observó que el PMP afectó el TC $(\mathrm{p}<0.05)$.

\section{Discusión}

Desde inicio de la formación de ambas líneas, la selección aplicada a los caracteres morfológicos externos como color de pelaje, color de ojos negros, tres dedos en las extremidades anteriores y cuatro en las posteriores presentan frecuencias relativas altas debido a la facilidad que representa el seleccio- nar caracteres controlados por pocos genes (Tave, 1996). Investigaciones locales revelan que las características externas de la línea Mantaro se han mantenido hasta la actualidad (Navarro, 2016; Reyes, 2015). Al evaluar la influencia de los caracteres morfológicos sobre los parámetros productivos y reproductivos, Reyes (2015) encontró diferencias significativas por efecto del año, pudiendo deberse a factores ajenos como el manejo, TC o NP (Rodríguez et al., 2013).

Los resultados para peso promedio al nacimiento de ambas líneas fueron superiores a lo reportado por Harkness et al. (2002) e INIA (2005) quienes mencionan pesos entre 45 y $115 \mathrm{~g}$, este último peso reportado 


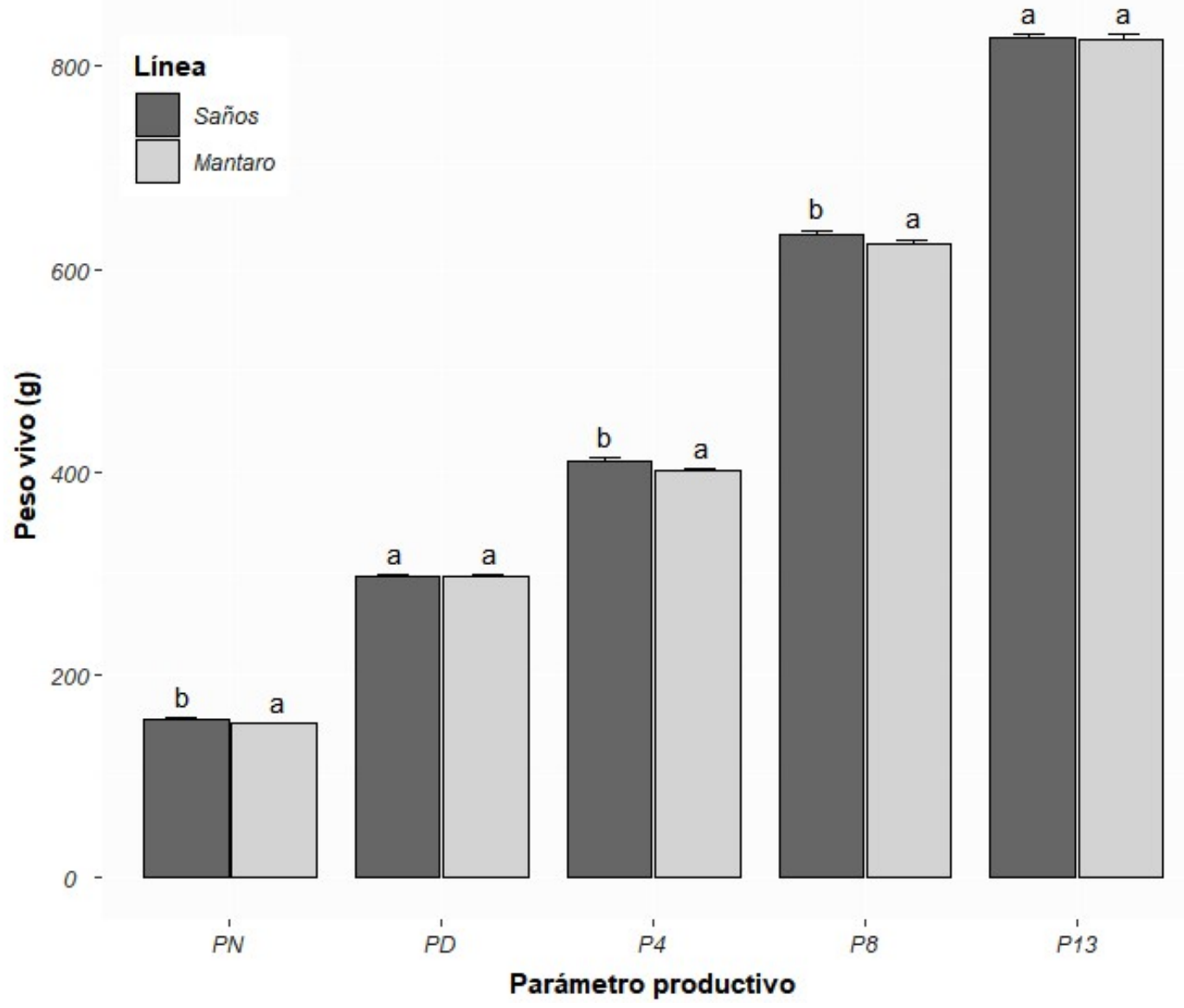

Figura 2. Medias mínimo cuadráticas de los parámetros productivos de peso al nacimiento (PN), peso al destete (PD), peso a la cuarta semana (P4), peso a la octava semana (P8) y peso a la décimo tercera semana (P13) para las líneas de cuyes Saños y Mantaro. Letras distintas para cada parámetro representan diferencias estadísticas $(\mathrm{p}<0.05)$

para la raza Andina. PN similares fueron reportados por Rodríguez et al. (2015) para el genotipo Cieneguilla $(146.9 \pm 33.5 \mathrm{~g})$, aunque inferiores a los $176 \mathrm{~g}$ para la raza Perú (INIA, 2011). Según Czarnecki y Adamski (2016), el PN depende en gran medida del $\mathrm{TC}$, enunciado que corrobora los resultados del presente estudio. Del mismo modo, la tendencia del PN a incrementar hasta el tercer parto y reducirse en partos posteriores podría estar relacionado con la madurez sexual y la longevidad
El PD según el número de parto varió entre $289.6 \pm 1.5$ y $299.4 \pm 6.4 \mathrm{~g}$, valores de menor variación que los descritos por Rodríguez et al., (2015) con valores mínimo y máximo de 265.6 y 322.2 g, respectivamente, posiblemente debido a la restricción alimentaria en madres y crías, instalaciones y temperatura, entre otros factores. LaurienKehnen y Trillmich (2004) y Kind et al. (2002) mencionan que, en el caso de restricciones alimentarias las madres normalmente mantienen su peso corporal, lo que conduce a dis- 
Cuadro 2. Medias mínimo cuadráticas \pm error estándar de pesos vivos (en gramos) de cuyes de las líneas Saños y Mantaro según el tamaño de camada, número de parto y sexo

\begin{tabular}{|c|c|c|c|c|}
\hline & \multicolumn{2}{|c|}{ Peso al nacimiento } & \multicolumn{2}{|c|}{ Peso al destete } \\
\hline & Saños & Mantaro & Saños & Mantaro \\
\hline \multicolumn{5}{|c|}{ Tamaño de camada } \\
\hline 1 & $188.0 \pm 2.0^{\mathrm{b}}$ & $184.7 \pm 2.0^{\mathrm{a}}$ & $302.6 \pm 4.2$ & $305.4 \pm 4.3$ \\
\hline 2 & $164.8 \pm 1.0^{\mathrm{b}}$ & $161.6 \pm 1.0^{\mathrm{a}}$ & $299.9 \pm 2.1$ & $302.6 \pm 2.1$ \\
\hline 3 & $146.5 \pm 0.9^{b}$ & $143.3 \pm 0.9^{\mathrm{a}}$ & $288.9 \pm 1.9$ & $291.6 \pm 1.9$ \\
\hline 4 & $135.0 \pm 1.1^{\mathrm{b}}$ & $131.8 \pm 1.3^{\mathrm{a}}$ & $284.0 \pm 2.6$ & $286.6 \pm 2.6$ \\
\hline 5 & $132.2 \pm 2.7^{\mathrm{b}}$ & $128.9 \pm 2.7^{\mathrm{a}}$ & $271.2 \pm 4.9$ & $273.8 \pm 5.0$ \\
\hline \multicolumn{5}{|c|}{ Número de parto } \\
\hline 1 & $150.6 \pm 1.0^{\mathrm{b}}$ & $147.4 \pm 1.9^{\mathrm{a}}$ & $284.5 \pm 1.8$ & $287.1 \pm 1.9$ \\
\hline 2 & $151.7 \pm 1.0^{\mathrm{b}}$ & $148.4 \pm 1.1^{\mathrm{a}}$ & $287.3 \pm 2.0$ & $290.0 \pm 2.0$ \\
\hline 3 & $157.6 \pm 1.2^{\mathrm{b}}$ & $154.3 \pm 1.2^{\mathrm{a}}$ & $293.5 \pm 2.3$ & $296.2 \pm 2.5$ \\
\hline 4 & $155.0 \pm 1.7^{\mathrm{b}}$ & $151.8 \pm 1.7^{\mathrm{a}}$ & $284.8 \pm 3.1$ & $287.4 \pm 3.4$ \\
\hline 5 & $151.6 \pm 3.0^{\mathrm{b}}$ & $148.4 \pm 3.0^{\mathrm{a}}$ & $295.6 \pm 6.3$ & $298.3 \pm 6.4$ \\
\hline \multicolumn{5}{|l|}{ Sexo } \\
\hline Hembra & $151.6 \pm 1.1^{\mathrm{b}}$ & $148.4 \pm 1.1^{\mathrm{a}}$ & $288.5 \pm 2.1$ & $291.2 \pm 2.2$ \\
\hline Macho & $155.0 \pm 1.1^{\mathrm{b}}$ & $151.8 \pm 1.1^{\mathrm{a}}$ & $289.7 \pm 2.2$ & $292.4 \pm 2.2$ \\
\hline
\end{tabular}

a,b Superíndices diferentes en la misma fila por parámetro productivo y edad indican diferencias significativas $(p<0.05)$

minuir el PN hasta en un $17 \%$, a un menor crecimiento postnatal y a un menor peso corporal en la madurez. Los estudios de Chauca (1997) e Higaonna Oshiro et al. (2004) reportaron pesos desde PN hasta P13; sin embargo, no consideraron los efectos fijos en su conjunto, de allí que no se podrían tomar como referencia.

En el cuy, como en muchas especies de interés zootécnico, los PMP influyen sobre el PN (Chauca, 1997; Rodríguez et al., 2013), lo que indica que reproductoras más pesadas dan crías ligeramente más pesadas al nacimiento. Del mismo modo, la significancia de las covariables evaluadas muestran que los animales con pesos evaluados en las etapas iniciales alcanzan mayores pesos en la etapa final, por el mismo hecho de que estos pesos están correlacionados a lo largo de la curva de crecimiento, tal como Coyne et al. (2016) observaron en porcinos.

Los cuyes se reproducen durante todo el año; sin embargo esto puede ser afectado principalmente por la disponibilidad y calidad de alimentos (Kind et al., 2003; LaurienKehnen y Trillmich, 2003, 2004). El TC suele ser entre 2 y 3.6 gazapos en promedio por camada con valores extremos que van de 1 a 7 gazapos (Rodríguez et al., 2015). El TC de la línea Mantaro en este estudio concuerda con lo reportado por Reyes (2015), quien da a conocer promedios no corregidos superiores a 2.5 gazapos. E1 TC de ambas líneas resultaron inferiores a los 3.21 y 3.20 gaza- 
Cuadro 3. Medias mínimo cuadráticas \pm error estándar de pesos vivos (en gramos) de cuyes de las líneas Saños y Mantaro según el tamaño de camada, número de parto y sexo

\begin{tabular}{ccccccc}
\hline & \multicolumn{2}{c}{ Semana 4} & \multicolumn{2}{c}{ Semana 8} & \multicolumn{2}{c}{ Semana 13 } \\
\cline { 2 - 7 } & Saños & Mantaro & Saños & Mantaro & Saños & Mantaro \\
\hline \multicolumn{2}{c}{ Tamaño de camada } & & & & & \\
1 & $422.9 \pm 3.2^{\mathrm{b}}$ & $416.6 \pm 3.3^{\mathrm{a}}$ & $646.0 \pm 8.5^{\mathrm{b}}$ & $634.9 \pm 8.5^{\mathrm{a}}$ & $832.7 \pm 6.1$ & $827.3 \pm 6.1$ \\
2 & $416.5 \pm 2.6^{\mathrm{b}}$ & $410.3 \pm 2.6^{\mathrm{a}}$ & $633.9 \pm 4.4^{\mathrm{b}}$ & $623.1 \pm 4.4^{\mathrm{a}}$ & $827.6 \pm 4.9$ & $822.1 \pm 5.0$ \\
3 & $410.2 \pm 2.5^{\mathrm{b}}$ & $404.1 \pm 2.9^{\mathrm{a}}$ & $622.5 \pm 4.2^{\mathrm{b}}$ & $611.8 \pm 4.2^{\mathrm{a}}$ & $822.4 \pm 4.7$ & $817.0 \pm 4.9$ \\
4 & $404.0 \pm 2.9^{\mathrm{b}}$ & $398.0 \pm 2.9^{\mathrm{a}}$ & $630.9 \pm 5.5^{\mathrm{b}}$ & $620.1 \pm 5.4^{\mathrm{a}}$ & $817.3 \pm 5.4$ & $811.9 \pm 5.6$ \\
5 & $397.9 \pm 3.7^{\mathrm{b}}$ & $392.0 \pm 3.7^{\mathrm{a}}$ & $616.5 \pm 6.8^{\mathrm{b}}$ & $606.0 \pm 6.8^{\mathrm{a}}$ & $812.2 \pm 6.8$ & $806.9 \pm 7.0$ \\
Número de parto & & & & & \\
1 & $408.8 \pm 2.4^{\mathrm{b}}$ & $402.4 \pm 2.4^{\mathrm{a}}$ & $618.9 \pm 3.6^{\mathrm{b}}$ & $608.3 \pm 3.8^{\mathrm{a}}$ & $833.7 \pm 3.5$ & $828.2 \pm 3.7$ \\
2 & $413.2 \pm 2.6^{\mathrm{b}}$ & $406.8 \pm 2.6^{\mathrm{a}}$ & $637.1 \pm 4.0^{\mathrm{b}}$ & $626.2 \pm 4.1^{\mathrm{a}}$ & $834.8 \pm 4.2$ & $829.4 \pm 4.5$ \\
3 & $408.3 \pm 3.0^{\mathrm{b}}$ & $401.9 \pm 3.1^{\mathrm{a}}$ & $621.6 \pm 4.2^{\mathrm{b}}$ & $611.0 \pm 4.9^{\mathrm{a}}$ & $824.5 \pm 5.1$ & $819.1 \pm 5.5$ \\
4 & $403.4 \pm 4.4^{\mathrm{b}}$ & $397.2 \pm 5.0^{\mathrm{a}}$ & $633.5 \pm 7.1^{\mathrm{b}}$ & $622.7 \pm 7.2^{\mathrm{a}}$ & $811.4 \pm 7.9$ & $806.0 \pm 8.1$ \\
5 & $404.5 \pm 9.4^{\mathrm{b}}$ & $398.3 \pm 9.2^{\mathrm{a}}$ & $638.6 \pm 14.8^{\mathrm{b}}$ & $627.6 \pm 14.5^{\mathrm{a}}$ & $813.7 \pm 16.9$ & $808.3 \pm 16.8$ \\
Sexo & & & & & & \\
H & $407.0 \pm 2.6^{\mathrm{b}}$ & $400.7 \pm 3.0^{\mathrm{a}}$ & $619.9 \pm 4.6^{\mathrm{b}}$ & $609.3 \pm 4.6^{\mathrm{a}}$ & $813.0 \pm 5.0$ & $807.7 \pm 5.1$ \\
M & $408.2 \pm 3.0^{\mathrm{b}}$ & $401.2 \pm 3.0^{\mathrm{a}}$ & $640.0 \pm 4.7^{\mathrm{b}}$ & $629.1 \pm 4.8^{\mathrm{a}}$ & $834.2 \pm 5.0$ & $828.8 \pm 5.2$ \\
\hline
\end{tabular}

a,b Superíndices diferentes en la misma fila por parámetro productivo y edad indican diferencias significativas $(p<0.05)$

pos reportado para el genotipo Cieneguilla y raza Andina, respectivamente; pero similar al TC de 2.61 de la raza Perú (INIA, 2005, 2011; Rodríguez et al., 2013), pero ciertamente medido bajo diferentes condiciones ambientales. Las dos líneas de este estudio se desarrollan en condiciones propias de la sierra central, distinta a las condiciones ambientales de la costa peruana. Por otro lado, los resultados muestran la influencia del PMP alimentadas ad libitum sobre el TC del parto siguiente; siendo las hembras con mayor PMP las que tienen menor TC; sin embargo, estas crías suelen ser más pesadas. Las hembras más pesadas almacenan grandes cantidades de tejido adiposo y muestran una tendencia a obesidad lo cual afecta negativamente la re-
Cuadro 4. Parámetros reproductivos de cuyes hembras de la línea Saños y Mantaro (Huancayo, Perú)

\begin{tabular}{lcc}
\hline & \multicolumn{1}{c}{ Saños } & Mantaro \\
\hline $\begin{array}{l}\text { Intervalo entre } \\
\text { partos (días) }\end{array}$ & $88.0 \pm 3.9^{\mathrm{a}}$ & $86.0 \pm 3.8^{\mathrm{a}}$ \\
$\begin{array}{l}\text { Tamaño de } \\
\text { camada }\end{array}$ & $2.60 \pm 0.05^{\mathrm{a}}$ & $2.64 \pm 0.04^{\mathrm{b}}$ \\
$\begin{array}{l}\text { a,b Superíndices diferentes } \\
\text { diferencias estadísticas }\end{array}$ & $\begin{array}{l}\text { representan } \\
\text { ( } \mathrm{p}<0.05)\end{array}$ \\
& &
\end{tabular}

producción (Michel y Bonnet, 2012; Czarnecki y Adamski, 2016). 
La fertilidad es un indicador de difícil medición en sistemas de empadre continuo como el de este estudio, por lo que autores como Cruz Flores (2015) y Pascual et al. (2017) proponen considerar el número de ciclos o partos al año en vez de la fertilidad. El número de ciclos va a depender del IP, pues a intervalos muy prolongados menor será la capacidad productiva de la granja. Ambas líneas no lograron superar los cinco ciclos por año (Quesenberry y Donnelly, 2019) y mucho menos al ideal (5.37 ciclos por año), considerando que la hembra es receptiva y fértil a las 3-4 horas posparto y presenta 68 días de gestación promedio (Pajares, 2009).

\section{Conclusiones}

- La capa de pelaje dentro de la línea de cuyes Saños y Mantaro está bien definida.

- El 97.75\% de cuyes de la línea Saños presenta un manto de coloración bayo o bayo con blanco grande en la cabeza.

- El 96.55\% de cuyes de la línea Mantaro tiene un color de pelaje predominante colorado con blanco grande o colorado con blanco pequeño en la cabeza

- Se han uniformizado caracteres como el número de dedos en las extremidades, coloración de ojos y presencia o ausencia de remolinos en el pelaje.

- El peso al nacimiento y a las 4 y 8 semanas de edad mostraron diferencias significativas, pero no biológicamente relevantes entre las dos líneas.

- El tamaño de camada fue mayor en la línea Mantaro, pero las diferencias no fueron relevantes.

- Los resultados muestran que no se ha logrado diferenciar las dos líneas por precocidad y prolificidad, como se venía asumiendo.

\section{Agradecimientos}

Trabajo financiado por el Instituto Interamericano de Cooperación para la Agricultura (IICA), mediante el proyecto «Mejora- miento de los Servicios Estratégicos de Innovación Agraria». Se agradece el apoyo de la Ing. Nancy Kajjak y Tec. Norma Garay Lazo (personales del INIA), quienes realizaron el trabajo de campo y generaron información desde el origen de la formación de ambas líneas de cuyes.

\section{Literatura Citada}

1. Arias RA, Mader TL, Escobar PC. 2008. Factores climáticos que afectan el desempeño productivo del ganado bovino de carne y leche Arch Med Vet 40: 7-22. doi: 10.4067/S0301-732X200800010000

2. Burgos-Paz W, Solarte-Portilla C, Cerón-Muñoz M. 2010. Efecto del tamaño de camada y número de parto en el crecimiento de cuyes (Cavia porcellus Rodentia: Caviidae). Rev Lasallista de Investigación 7: 47-55.

3. Chauca L. 1997. Producción de cuyes (Cavia porcellus). FAO. http://www.fao.org/3/w6562s/w6562s00.htm\#TopOfPage

4. Coyne JM, Matilainen D, Berry DP, Sevon-Aimonen ML, Mäntysaari EA, Juga J, Serenius T. 2016. Estimation of genetic (co) variances of Gompertz growth function parameters in pigs. $\mathrm{J}$ Anim Breed Genet. doi: 10.1111/ jbg. 12237

5. Cruz Flores DJ. 2015. Estimación de pesos económicos en la producción intensiva de cuyes (Cavia porcellus) productores de carne. Tesis de Maestría. Valencia, España: Universidad Politécnica de Valencia. $50 \mathrm{p}$.

6. Czarnecki R, Adamski M. 2016. Factors influencing litter size and birthweight in the newborn long-haired guinea pigs (Cavia aperea f. porcellus). J Appl Anim Res 44: 71-76. doi: 10.1080/ 09712119.2015.1013961

7. Dillard EU, Vaccaro R, Lozano J, Robison OW. 1972. Phenotypic and genetic parameters for growth in guinea 
pigs. J Anim Sci 34: 193-195. doi:10.2527/jas1972.342193x

8. Harkness JE, Murray KA, Wagner JE. 2002. Biology and diseases of amphibians. In: Laboratory animal medicine. Elsevier. p 203-246).

9. Higaonna $R$, Muscari J, Chauca L. 2004. Evaluación del cruzamiento del cuy de la raza Perú con Merino. En: XXVII Reunión de la Asociación Peruana de Producción Animal. Lima, Perú.

10. INEI. 2018. Encuesta Nacional Agropecuaria 2017. Principales Resultados. Pequeñas, medianas y grandes unidades agropecuarias. https://www.inei.gob.pe/ media/MenuRecursivo/publicaciones_digitales/Est/Lib1593/

11. INIA. 2005. Cuy raza Andina. https:// www.inia.gob.pe/wp-content/uploads/ investigacion/programa/sistProductivo/ raza/cuy/Cuy-raza-andina.pdf

12. INIA. 2011. Cuy raza Perú. https:// www.inia.gob.pe/wp-content/uploads/ investigacion/programa/sistProductivo/ raza/cuy/Cuy-raza-peru.pdf

13. INKACUY. 2018. Junín: nacen dos futuras líneas de cuyes, Mantaro y Saños - Inka Cuy. [Internet]. Disponible en: http://www.inkacuy.com.pe/junin-nacendos-futuras-lineas-de-cuyes-mantaro-ysanos

14. Kind KL, Clifton PM, Grant PA, Owens PC, Sohlstrom A, Roberts CT, Robinson JS, et al. 2003. Effect of maternal feed restriction during pregnancy on glucose tolerance in the adult guinea pig. Am J Physiol 284: R140-52. doi: 10.1152/ajpregu.00587.2001

15. Kind, KL, Simonetta G, Clifton PM, Robinson JS, Owens JA. 2002. Effect of maternal feed restriction on blood pressure in the adult guinea pig. Exp Physiol 87: 469-477. doi: 10.1111/j.1469445X.2002.tb00060.x

16. Laurien-Kehnen C, Trillmich F. 2003. Lactation performance of guinea pigs (Cavia porcellus) does not respond to experimental manipulation of pup demands. Behav Ecol Sociobiol 53: 145152. doi: $10.1007 / \mathrm{s} 00265-002-0550-7$

17. Laurien-Kehnen C, Trillmich F. 2004. Maternal food restriction delays weaning in the guinea pig, Cavia porcellus. Anim Behav 68: 303-312. Doi: 10.1016/ j.anbehav.2003.11.012

18. Lobo I. 2008. Environmental influences on gene expression. Nature Educ. [Internet]. Available in: https://www.-nature.com/ scitable/topicpage/environ-mentalinfluences-on-gene-expression-536/

19. Michel CL, Bonnet X. 2012. Influence of body condition on reproductive output in the guinea pig. J Exp Zool Part A: Ecol Genetics Physiol 317A: 24-31. doi: $10.1002 /$ jez. 714

20. Navarro JE. 2016. Caracterización zoométrica de cuyes de la Línea Mantaro en la E.E.A. Santa Ana - INIA Huancayo. Tesis de Ingeniero Zootecnista. Huancayo, Perú: Univ. Nacional del Centro del Peeú. $72 \mathrm{p}$.

21. Pajares C. 2009. Reproducción y manejo reproductivo en cuyes (Cavia porcellus). Lima, Perú. Univ Nacional Mayor de San Marcos. 8 p.

22. Pascual M, Cruz DJ, Blasco A. 2017. Modeling production functions and economic weights in intensive meat production of guinea pigs. Trop Anim Health Prod 49: 1361-1367. doi: 10.1007/ s11250-017-1334-4

23. Quesenberry KE, Donnelly TM. 2019. Breeding and reproduction of guinea pigs. In: MSD Manual. Veterinary Manual. [Internet]. Available in: https://www.msdvetmanual.com/all-other-pets/guinea-pigs/breeding-and-reproduction-ofguinea-pigs

24. Quijandria B, Chauca L, Robison OW. 1983. Selection in guinea pigs: I. Estimation of phenotypic and genetic parameters for litter size and body weight. J Anim Sci 56: 814-819. doi: 10.2527/jas1983.564814x 
25. $R$ Core Team. 2020. R: A language and environment for statistical computing. [Internet]. Available in: https://www.rproject.org/

26. Reyes P. 2015. Influencia de las características fenotípicas externas de cuyes de la línea Mantaro en la producción y reproducción, en la Estación Experimental Santa Ana del INIA - Huancayo. Tesis de Ingeniero ootecnista. Huancayo, Perú: Univ. Nacional del Centro del Perú. 67 p.

27. Rodríguez H, Gutiérrez G, Palomino M, Hidalgo V. 2015. Características maternales al nacimiento y destete en cuyes de la costa central del Perú. Rev Inv Vet Perú 26: 77-85. doi: 10.15381/ rivep.v26i1.10941
28. Rodríguez H, Palomino M, Hidalgo V, Gutiérrez G. 2013. Efectos de factores fijos y al azar sobre el peso al nacimiento y al destete en cuyes de la costa central del Perú. Rev Inv Vet Perú 24: 16-24. doi: 10.15381/rivep.v24i1.1647

29. Sánchez-Macías D, Barba-Maggi L, Morales-de la Nuez A, Palmay-Paredes J. 2018. Guinea pig for meat production: a systematic review of factors affecting the production, carcass and meat quality. Meat Sci 143: 165-176. doi: 10.1016/j.meatsci.2018.05.004

30. Tave D. 1996. Programas de cría selectiva para piscifactorías de tamaño medio. FAO Documento Técnico de Pesca 32. Roma: FAO. [Internet]. Disponible en: http://www.fao.org/3/v8720s/ v8720s02.htm 